\title{
Efecto de la certificación docente en el cambio de las prácticas pedagógicas. Un análisis documental
}

\section{The effect of teacher certification on changes in pedagogical practice: a documentary analysis}

DOI: $10.32870 /$ dse.v0i19.501

\author{
Óscar Zaragoza-Vega* \\ Martha Patricia Gutiérrez-Pérez**
}

\begin{abstract}
Resumen
Propósito: A partir de la revisión de la literatura, se identifican aspectos conceptuales de las innovaciones de la práctica docente, que han permitido comprender y reflexionar sobre la transcendencia de los procesos formativos y de convalidación del logro de las competencias docentes. Metodología: se realizó una revisión documental para identificar conceptos sobre la innovación y transformación de las prácticas educativas, así como los posibles efectos de la formación y la certificación docente en la calidad del aprendizaje. Principales resultados: La innovación docente implica que los profesores adopten una forma distinta de trabajo mediante la incorporación de nuevos elementos a su práctica, y los trabajos analizados indican que existe clara tendencia a incorporar la TIC como elemento innovador. Asimismo, la transformación permanente de estas prácticas es posible mediante la reflexión y los procesos de evaluación del desempeño, siempre y cuando se considere la retroalimentación. Discusión: Las demandas educativas actuales y futuras exigen el dominio de competencias de los profesores para utilizar herramientas de la era digital y transformar su práctica, lo cual es posible a través de procesos reflexivos, que permiten al docente consolidar sus metodologías y fortalecer su propio aprendizaje; la evaluación del desempeño docente debe centrarse en el desempeño del profesor, que posibilite la recuperación de información valiosa sobre su actuación así como obtener retroalimentación para promover la reflexión. No se encontraron trabajos que relacionen la efectividad en los resultados de aprendizaje ligados a la certificación docente.
\end{abstract}

Palabras clave: Innovación docente - Transformación de la práctica - Evaluación y certificación

\begin{abstract}
Purpose: Based on the literature, conceptual aspects of innovations in teaching practices were identified in order to understand and reflect on the transcendence of the formative processes, and confirm the achievement of teaching competences. Methodology: a documentary review was conducted to identify concepts
\end{abstract}

* Maestro en Enseñanza de las Ciencias. Consultor ambiental, consultor para la actualización de programas de las Unidades de Aprendizaje. Responsable del área de investigación de la Dirección de Formación Docente e Investigación del Sistema de Educación Media Superior, Universidad de Guadalajara, México. oscar.zaragoza@sems.udg.mx

** Maestra en Enseñanza de las Ciencias. Consultora para la actualización de programas de las Unidades de Aprendizaje en nivel medio y superior en diferentes dependencias de la Universidad de Guadalajara. Formadora Docente a nivel nacional. México. gupmpaty@gmail.com 
about innovation and transformation of educational practices, as well as the possible impact of teacher training and certification on the quality of learning. Main results: Innovation in teaching implies adopting different ways of working by incorporating new components into the teacher's practice, and the papers analyzed show that there is a clear tendency to incorporate ICT as an innovative element. Likewise, a permanent transformation of these practices can only be achieved through reflection and performance evaluation processes, provided that feedback is taken into account. Discussion: Present and future educational demands require the training of teachers to use tools of the digital age and transform their practice, which is possible only through thorough reflection that allows teachers to consolidate their methodologies and reinforce their own learning. Teaching performance evaluation should focus on the teacher's actual performance, retrieving valuable information about their performance and at the same time providing feedback to promote reflection. No studies were found that relate the effectiveness in the results of learning linked to teacher certification.

Key words: Teaching innovation - Practice transformation - Assessment and certification

\section{Introducción}

Desde el origen de la reforma educativa del nivel medio superior (RIEMS) en el año de 2008, las opciones de certificación han tenido el carácter de autogestoras, y desde su origen se ha esperado que el profesor acuda a las convocatorias emitidas. Uno de los procesos de certificación que logró mayor efecto en los profesores del Sistema de Educación Media Superior en la Universidad de Guadalajara ha sido, sin duda, el CERTIDEMS-ANUIES, proceso que en su primera etapa ofrecía cinco opciones de certificación, de las cuales tres estaban dirigidas a docentes que habían cursado el Diplomado en Competencias Docentes para la Educación Media Superior, mientras que una era orientada a directivos que cursaron el Diplomado de Competencias Directivas (PROFORDIR) y una para docentes que hubiesen cursado alguna de las Especialidades para el Nivel Medio Superior de la Universidad Pedagógica Nacional (UPN).

Por otra parte, en 2011 se instituye el Consejo Técnico del Proceso de Evaluación de Competencias Docentes para Educación Media Superior (ECODEMS), del Centro Nacional de Evaluación Para la Educación Superior, A.C. (CENEVAL). Mismo que convoca desde entonces al proceso para obtener una certificación mediante: una prueba de conocimientos, una planeación didáctica, una prueba de desempeño grabada en video y una reflexión escrita.

En el año 2015 se inició el proceso para ingreso, permanencia o promoción en el sistema educativo mexicano, procedimiento establecido en la Ley del Servicio Profesional Docente, mediante el cual se convoca a los docentes a un proceso de evaluación del desempeño docente.

Asimismo, el Fideicomiso de los Sistemas Normalizados de Competencia Laboral y de Certificación de Competencia Laboral (CONOCER), ofrece el Estándar de Competencia EC0646 para la certificación, y Facilitación de Procesos de Aprendizaje con el enfoque de Competencias en Educación Media Superior. 
La Certificación de Competencias Docentes para la Educación Media Superior tiene como propósito validar la aplicación de competencias del profesor desde un enfoque formativo centrado en el aprendizaje, así como verificar las innovaciones y transformaciones en su propia práctica docente. En ambos procedimientos se busca evidenciar que el docente ha incorporado innovaciones y transformaciones a su práctica educativa como consecuencia de un proceso de formación y actualización profesional.

La innovación es un factor relevante que moviliza cambios pertinentes con fines de mejora de la praxis del docente (Harvey, 2015), es decir, implica que el profesor debe realizar intervenciones sobre su actuación, a fin de modificar sus estrategias de trabajo en el aula a partir de la reflexión sobre su propio proceso de aprendizaje (Santiago, Navaridas y Andía, 2016).

Para Rosario, Elicerio, Zaragoza, Varela y Partida (2016) la innovación de la práctica docente es un proceso permanente y continuo, mediante el cual actualiza y proporciona intención a sus acciones, a fin de mejorar los resultados de aprendizaje de sus alumnos y, como consecuencia de estas modificaciones, se transforma la práctica docente.

Desde el inicio de la Reforma Integral de la Educación Media Superior (RIEMS, 2008) se ha incrementado el número de docentes certificados en competencias. Sin embargo, no hay evidencia sobre las innovaciones y/o transformaciones que han puesto en juego en el aula, que evidencien el logro de las competencias docentes esperadas. Se reconoce que uno de los principales retos sobre la evaluación de competencias no estriba en los instrumentos, sino en los escenarios mediante los cuales se pueda evidenciar de manera objetiva el desempeño esperado de las personas para la resolución de problemas contextualizados (Hernández, 2013).

Varios estudios han encontrado que los maestros no cambian fácilmente sus concepciones, y menos aún sus prácticas educativas que, dependiendo del maestro y el contexto, las concepciones y las prácticas a menudo están desfasadas unas de otras, e incluso claramente en contradicción -especialmente para profesores principiantes-, y que los cambios en uno no están necesariamente acompañados por un cambio en el otro (Vázquez-Bernal, Mellado, Jiménez-Pérez y Taboada, 2011).

Por lo anterior, se hace necesario establecer conceptualmente en qué consisten las innovaciones y transformaciones de la práctica docente, para generar acuerdos en la comunidad académica, en particular, de los docentes certificados del Sistema de Educación Media Superior de la Universidad de Guadalajara, a fin de perfilar indicadores para la toma de decisiones en torno a los procesos de consolidación de la enseñanza centrada en el estudiante.

Por su parte, la innovación no puede existir sin el individuo que se compromete con ideas y conceptos para promover sus capacidades a fin de introducir cambios en su actuación cotidiana, y donde el docente es el principal responsable de efectuar estos cambios para procurar la mejora de los resultados escolares (Marcelo, 2013; Álvarez, 2015).

Existe consenso respecto al enfoque de la formación de docentes en la actualidad, cuyo abordaje se centra en que los profesores incorporen nuevas estrategias y técnicas que respon- 
dan a la sociedad de la información y la comunicación (Muñoz, Serrano y Marín, 2014) y que la reflexión sistemática sobre las prácticas docentes sobre el aprendizaje requiere un profundo análisis de las fortalezas y áreas de mejora para lograr la transformación del trabajo docente. Asimismo, se admite que para consolidar un sistema educativo diferente e innovador es necesario hacer énfasis en los procedimientos, técnicas y métodos que utiliza el docente (Padilla, 2014).

Para Ruiz (2013), la vía con mayores posibilidades de éxito es la capacitación de los docentes a partir de la reflexión sobre su propia práctica, lo que debe resultar en un proceso de enriquecimiento y cambio en la actuación, y de crecimiento personal para comprenderse desde dentro ya que se parte de la identificación de las motivaciones (pensamientos, sentimientos e intenciones).

El proceso reflexivo que hace un docente sobre su propia práctica, considerando, por una parte, los componentes de enseñanza, y por otra, la concepción que se tiene sobre el aprendizaje, son el punto de partida para identificar sus fortalezas, así como para clarificar las áreas de mejora (Muñoz, Serrano y Marín, 2014); este parece ser un principio fundamental para promover el cambio en las prácticas pedagógicas. Por ello, es fundamental explorar en qué medida los docentes que han transitado por procesos formativos y de certificación realizan este ejercicio y proponen incorporar elementos de innovación.

Según Rosario (2016), el cambio de la práctica docente se provoca por el diseño de la innovación con base en el criterio de vincular la teoría con la práctica mediante el proceso de problematizar el acto educativo, reconociendo las acciones educativas derivadas de la práctica para resignificar las acciones educativas. Esta resignificación produce como consecuencia la transformación, misma que se consolida mediante la recuperación y análisis sobre las intervenciones e interacciones del acto educativo.

En relación con la planificación didáctica, se reconoce puntualmente que es importante poner en práctica las habilidades y capacidades de los docentes, para planear, diseñar e implementar secuencias de aprendizaje para el desarrollo de competencias en sus estudiantes (Herrera-Sánchez, 2017; González-Bernal, 2016). En este sentido, el docente debe poseer la capacidad de seleccionar, elaborar, adaptar y programar recursos didácticos, materiales y ambientes favorables para el aprendizaje, así como de llevar a cabo el proceso de evaluación de manera continua (González-Olivares, 2014).

Durante la planificación se hace necesario que los profesores reflexionen de manera profunda y explícita sobre las competencias que ellos mismos poseen y las que pretenden desarrollar en sus estudiantes, lo que a su vez debe permitir identificar, a partir de la evaluación, niveles de logro (Hernández-Jaime, 2015).

Ante los nuevos escenarios, se espera que los docentes demuestren un amplio nivel de desempeño, en donde la complejidad del proceso de enseñanza-aprendizaje implica que el do-

Diólo pos año 10 | número 19 | julio-diciembre 2019 | ISSN 2007-2171 
cente sea capaz de controlar un conjunto de variables tales como: las metodologías centradas en el estudiante, las técnicas para promover diferentes aprendizaje (conocimientos, habilidades y actitudes), y aplicar procesos de evaluación continua. De igual manera, y ante los nuevos escenarios de la era de la información y comunicación, la atención a los estudiantes implica el diseño de estrategias mediadas por las tecnologías de la información y comunicación, herramientas de la Web como: blogs, plataformas educativas, aplicaciones para móviles, solo por mencionar algunas.

De acuerdo con lo expuesto, el presente estudio conceptual se enfoca en las siguientes metas: analizar el concepto de innovación y transformación de la práctica didáctica con base en la bibliografía actual; establecer los logros y dificultades en la transformación de las prácticas pedagógicas; y analizar el posible efecto de los procesos de certificación docente en la mejora e innovación de las prácticas pedagógicas.

Finalmente, la principal aportación de este trabajo es el análisis exhaustivo sobre indicadores conceptuales de las innovaciones de la práctica del profesor mediante el análisis documental, que permitan comprender y reflexionar sobre la trascendencia de los procesos formativos y de convalidación del logro de las competencias docentes.

\section{Metodología}

\section{Tipo de estudio}

El análisis documental, como procedimiento científico, consiste en el acceso a fuentes de información, y se realiza aplicando ciertos métodos sistemáticos para la recuperación de los datos (Peña, 2007). Para este trabajo, la búsqueda tuvo dos propósitos básicos: 1) explorar las aportaciones, y 2) identificar conceptos de diversos autores sobre la innovación y transformación de las prácticas educativas aportados en los últimos años, e identificar su relación con procesos de certificación de competencias docentes en el marco de la Reforma Integral de la Educación Media Superior en México.

Para Ortega-Carbajal, Hernández-Mosqueda y Tobón-Tobón (2015), y Hernández-Mosqueda, Guerrero-Rosas y Tobón-Tobón (2015), el análisis documental se realiza de manera profesional y científica cuando se llevan a cabo procesos de búsqueda, recuperación y análisis de información que ha sido compartida por otros autores mediante artículos, y existen al menos cinco etapas para lograrlo: la primera, realizar búsquedas de información confiable relacionadas con el tema de investigación; la segunda, recuperar y analizar los textos con la finalidad de establecer categorías; la tercera, organizar la información de acuerdo con las categorías; cuarta, análisis crítico de la información en relación con el tema de investigación; y quinta, detectar vacíos a fin de hacer propuestas. 


\section{Categorías de análisis}

Para este trabajo, la recuperación y análisis documental se realizó mediante la búsqueda y selección de artículos en revistas indexadas, relacionados con los procesos de certificación docente y de innovación de la práctica educativa, y la planificación del proceso de enseñanza-aprendizaje; esto permitió realizar un registro bibliográfico de fuentes primarias. Se seleccionaron prioritariamente artículos de los últimos cuatro años; sin embargo, resultó necesario incluir algunos de mayor antigüedad debido a que no se encontró material de años recientes. Las categorías analíticas establecidas se observan en la tabla 1.

Tabla 1. Análisis de categorías empleadas en el estudio

\begin{tabular}{|l|l|}
\hline \multicolumn{1}{|c|}{ Categorías } & \multicolumn{1}{|c|}{ Preguntas o componentes } \\
\hline $\begin{array}{l}\text { Conceptualización sobre la } \\
\text { innovación docente }\end{array}$ & $\begin{array}{l}\text { Concepto de innovación didáctica o innovación } \\
\text { docente }\end{array}$ \\
\hline & $\begin{array}{l}\text { Componentes que caracterizan la innovación } \\
\text { docente }\end{array}$ \\
\hline $\begin{array}{l}\text { Conceptualización de transfor- } \\
\text { mación docente }\end{array}$ & $\begin{array}{l}\text { Concepto de transformación de la práctica do- } \\
\text { cente } \\
\text { de la práctica }\end{array}$ \\
\hline & $\begin{array}{l}\text { Diferencias entre innovación y transformación } \\
\text { de la práctica docente }\end{array}$ \\
\hline $\begin{array}{l}\text { Evaluación de competencias y } \\
\text { el desempeño desde la socio- } \\
\text { formación }\end{array}$ & $\begin{array}{l}\text { Concepto de evaluación desde la sociofor- } \\
\text { mación }\end{array}$ \\
\hline Sobre la certificación docente & Componentes de una evaluación socioformativa \\
\hline & Concepto de certificación docente \\
\hline & $\begin{array}{l}\text { Certificación de docentes y su relación con la } \\
\text { efectividad del aprendizaje }\end{array}$ \\
\hline
\end{tabular}

Fuente: Elaboración propia.

\section{Criterios de selección de los documentos}

La selección y recuperación de la información, aplicadas para la realización de este artículo, se explican a continuación:

1. Búsqueda de artículos y libros a través de las bases de datos: Google Académico, WoS, Science Direct, Scielo, Redalyc y Latindex.

2. Las palabras esenciales aplicadas en la búsqueda fueron las siguientes "innovación didáctica", "transformación y práctica docente", "planificación didáctica"; junto con una o 
varias de las siguientes palabras complementarias: "innovación de la práctica docente", "transformación y didáctica", "planeación docente" y"certificación docente".

3. La selección de los artículos se realizó en función de que fueran exclusivamente artículos de revistas indexadas. Sin embargo, fue necesario seleccionar libros de editoriales reconocidas, centros de investigación o universidades.

4. Los textos debían estar dentro del periodo 2014-2017; sin embargo, fue necesario incorporar algunas referencias de mayor antigüedad.

5. Los documentos debían abordar algún elemento de las categorías establecidas.

\section{Documentos analizados}

A continuación, se presenta una síntesis cuantitativa de los textos seleccionados para este artículo, donde se contabiliza un total de 18 textos recuperados; de ellos, tres fueron considerados para contextualizar y complementar el análisis (tabla 2).

Tabla 2. Documentos analizados en el estudio

\begin{tabular}{|c|c|c|c|c|}
\hline Documentos & Sobre el tema & $\begin{array}{c}\text { De contex- } \\
\text { tualización o } \\
\text { complemento }\end{array}$ & $\begin{array}{c}\text { Latinoamerica- } \\
\text { nos }\end{array}$ & $\begin{array}{c}\text { De otras re- } \\
\text { giones }\end{array}$ \\
\hline Artículos teóricos & 7 & 2 & 8 & 1 \\
\hline Artículos empíricos & 43 & 0 & 20 & 20 \\
\hline Libros & 1 & 0 & 1 & 0 \\
\hline Informe & 0 & 1 & 1 & 0 \\
\hline
\end{tabular}

Fuente: Elaboración propia.

\section{Resultados}

\section{Categoría 1. Conceptualización sobre la innovación docente}

A continuación, se describen los resultados de la búsqueda de información relacionada con los conceptos de innovación docente. A últimas fechas, se reconoce en nuestro país que el éxito o el fracaso escolar es debido al trabajo de los docentes; en este sentido, se ha insistido en las políticas nacionales en dos grandes vertientes: la formación y la profesionalización. En el sentido de la formación, se busca que los docentes incorporen una renovada metodología de trabajo, en donde abandonen su papel estelar y se conviertan en facilitadores de procesos de aprendizaje. En este cambio metodológico se espera su profesionalización y, por lo tanto, un giro en las relaciones entre los aprendizajes esperados, los contenidos, el docente y los estudiantes (Romero, 2017).

La innovación docente implica que los profesores adopten una forma distinta de trabajo incorporando nuevos componentes a su práctica, cambiar la forma de planificar el aprendizaje, así como incluir técnicas, estrategias, formas de interactuar y guiar a los estudiantes. En este 
sentido, León-Guerrero (2014) señala que no se puede hablar de innovación docente sin buenas prácticas, en las que el docente es responsable de establecer los aprendizajes esperados, así como las acciones que sus estudiantes deben realizar para lograr las metas cuya medición se evidencia con los indicadores escolares, para verificar la efectividad y eficacia de las acciones de innovación docente.

Con respecto a lo anterior, Ausín (2016) reporta una experiencia de innovación docente con la aplicación del ABP (aprendizaje basado en problemas) mediante la incorporación de las tecnologías de información y comunicación (TIC), y señala que esta forma de trabajo orienta al docente y a los estudiantes hacia un trabajo productivo y alejado de las acciones de repetición y memorización del estilo docente tradicional, donde se manifiesten la autonomía, el trabajo grupal, mayor confianza y capacidad para enfrentar problemas de la realidad.

La incorporación de las TIC como herramienta para la innovación es con mucha frecuencia sinónimo de innovación docente, en donde destacan las plataformas educativas como una forma diferente para relacionarse y comunicarse con sus profesores y el aprendizaje (Olmos Alcaraz, 2015) o, como lo señala Vera (2014), lo fundamental de incorporar las TIC como parte de las nuevas estrategias de enseñanza-aprendizaje es promover en los profesores nuevas capacidades para propiciar el aprendizaje.

Maroto (2017) refiere que la forma de trabajo docente mediante el método de caso, es también una estrategia de innovación de la práctica docente ya que permite a los estudiantes el acercamiento con situaciones de aprendizaje a partir de problemas de la realidad, lo que implica promover sus saberes e involucrarse en distintas formas de solución, ya sea de forma individual como en el trabajo en grupo, y agrega que esta metodología favorece habilidades que de otra forma no podrían observarse en los estudiantes, como el desarrollo del espíritu crítico y el ingenio.

Para Medina (2015), la innovación docente debe involucrar al menos tres grandes dimensiones; a) el contenido, b) el conocimiento pedagógico y c) tecnología (TIC), en las que los profesores deben desarrollar competencias para la creación de: blogs, páginas interactivas, materiales educativos a través de la red, tutoriales, guías, trabajos monográficos, cursos, entre otros; y precisan que las metodologías son los objetos de la innovación cuya idea es desarrollar un modo de trabajar distinto, donde concurren elementos distintos a lo antes hecho, mediante la creatividad, una cuidadosa selección de contenidos y materiales. Algunas de las innovaciones en las prácticas docentes resaltan el uso del portafolio digital como una estrategia que facilita los procesos de enseñanza-aprendizaje (López, 2013).

Entre otras de las nuevas formas de trabajo docente encontradas, y que muestran innovación docente, están el aprendizaje mediante las visitas a centros de trabajo, lo que promueve en los estudiantes la imaginación y la proyección de un futuro cercano, haciendo evidentes posibles opciones de desarrollo profesional (Muñoz, 2017). 
Por otra parte, esta nueva docencia no solo debe enfocar los esfuerzos formativos de las personas en saber hacer, sino desarrollar procesos formativos con carácter humanista, haciendo énfasis en el fortalecimiento de los valores y las actitudes para enfrentar los nuevos retos sociales; esto implica que la innovación docente debe incorporar así una dimensión para la formación de personas con integralidad (Seixas, 2016).

Otra metodología reconocida por la alta participación de los estudiantes y como innovación docente, señalada por Pérez (2017), es el trabajo mediante el uso de mandos interactivos de respuesta rápida (clickers), que implica una delicada planeación por parte del profesor, a fin de crear elementos de interacción dentro del aula, ya sea mediante preguntas, imágenes $u$ otros contenidos.

Se observa entonces que la innovación docente implica una transición, de un modelo educativo centrado en la enseñanza a uno centrado en el aprendizaje, donde cobran importancia las Ilamadas metodologías activas, que parecen ser las más adecuadas para fomentar el trabajo colaborativo, organizar la enseñanza basada en competencias y estimular la adquisición de aprendizajes autónomos y permanentes (Crisol, 2017).

En algunos trabajos sobre innovación se reconoce la importancia de las metodologías activas que permiten al docente ser claro y preciso en sus explicaciones, motivadoras, que inducen a la reflexión y la autocrítica, así como otras habilidades que ya se han mencionado antes (Bueno, 2017).

\section{Categoría 2. Conceptualización de transformación docente}

Una vez aclarado que la innovación se refiere a la introducción de cambios en las metodologías de trabajo de los profesores, la transformación docente implica, según Cela-Ranilla (2017), la integración de procesos reflexivos que favorecen las dinámicas entre estudiantes y docentes en diálogo compartido, por lo que se aprende en un contexto de real conciencia que favorece el crecimiento de las personas.

Por otra parte, se dice que la transformación de la práctica docente solo se genera si los propios sujetos (docentes) se convierten en el objeto de investigación, identificando, mediante la conceptualización propia de la actividad docente, reflexionando de manera analítica la práctica educativa como una actividad dinámica, sistemática, reflexiva, la cual ocurre antes, entre y luego de cualquier planificación que haga el docente como productor, forjador y formador. De esta manera, el docente y sus metodologías se consolidan (Margelis, 2017).

Para Morán (2017), una herramienta útil en la promoción de reflexión sobre el trabajo de los profesores y la transformación de la práctica, es la evaluación del desempeño que permite recuperar información para desarrollar un análisis y transformación de la práctica docente propia. Este proceso evaluativo debe regular los procesos de enseñanza-aprendizaje y orientar la dirección de sucesivos procesos para conocer la eficacia de las actividades realizadas y determinar el grado de éxito en relación con los objetivos que se persiguen. 
Para consolidar los aprendizajes del docente sobre su propio actuar, es importante que se identifiquen los diferentes componentes que utiliza en su trabajo, y estas fuentes de información vienen de los aportes entre colegas a través de la coevaluación, así como de la autoevaluación y la heteroevaluación proveniente de estudiantes y directivos (González, 2017). De esta manera se contribuye a visibilizar los saberes del profesor y mediante la reflexión para la construcción y deconstrucción del proceso de enseñanza-aprendizaje (Pierella, 2017).

Los procesos formativos de los profesores deben entonces fomentar el aprendizaje individual, generar una visión interna del proceder y actuar docente, problematizar e investigar sobre su práctica, y acompañarse de los demás con la finalidad de afianzar su propio aprendizaje (Veloquio, 2017). Así, las habilidades y competencias en la docencia se configuran en la transformación, es decir, en un aprendizaje a largo plazo, duradero y consolidado, que permita a su vez iniciar un nuevo ciclo de innovación-transformación.

Según Figueroa (2017), los profesionales de la enseñanza deben reflexionar sobre diferentes dimensiones de la práctica docente, sobre los contenidos que desea enseñar, las estrategias con las que pretende promover el aprendizaje, la estructura y el nivel del currículo que le fue asignado, los aprendizajes esperados o competencias a observar, así como los fundamentos del aprendizaje y la filosofía con la que se promueven, las consideraciones sobre la realidad contextual propia y de sus estudiantes. Al visualizar este marco referencial, el docente puede entonces elaborar una representación de su identidad docente.

Estas dimensiones del ser docente deben ser ordenadas, estructuradas y externadas para ser apropiadas, y así valorar su pertinencia como pilares de la práctica didáctica. Al recrearlas a través de la narrativa del sujeto, se crea la propia historia docente y se entiende su transformación (Suárez, 2017); así, el aprendizaje sobre la enseñanza y la enseñanza de la enseñanza, involucran la adquisición y el desarrollo de una amplia serie de habilidades, conocimientos y competencias del docente y apuntalan el crecimiento profesional (Fardiño, 2017).

Sobre la idea de profesionalización docente, Ávila-Meléndez (2017) señala que los docentes deben afrontar la formación como la oportunidad de ejercitar sus habilidades, de ejercer un rol activo y su creatividad, que implican nuevas formas de solucionar los problemas de la docencia en un ejercicio teórico-práctico para lograr mejores desempeños y, sobre todo, reflexionar a lo largo de esta ruta.

\section{Categoría 3. Evaluación de competencias y el desempeño desde la socioformación}

El tema de la evaluación docente ha cobrado especial relevancia en nuestro país en los últimos años, entre otras cosas, debido a las políticas institucionales establecidas por la Ley del Servicio Profesional Docente; y en este escenario se han propuesto novedosas estrategias basadas en instrumentos (rúbricas, listas de cotejo, portafolios) que permiten valorar evidencias que producen los docentes y reconocer su desempeño frente a grupo, así como favorecer los procesos

Diálo@os sobre Educación año 10 | número 19 | julio-diciembre 2019 | ISSN 2007-2171 
de transformación de la práctica (Hernández-Mosqueda, 2016). En este contexto, para Leyva (2017), los procesos evaluativos en el nuevo modelo educativo, tanto para el docente como para los estudiantes, son procesos que permiten generar información que puede ser recuperada para procesarla, organizarla e interpretarla, a fin de identificar las transformaciones y el logro de las habilidades integrales del ser humano.

\section{Categoría 4. Sobre la certificación docente}

La innovación y la transformación deben reflejarse de manera consistente, por lo que surgen los procesos de certificación: se trata de convalidar la innovación y la transformación de la práctica docente (Oviedo, 2016). En un análisis sobre los esfuerzos de la Universidad Autónoma del Carmen, los autores Herrera, Sánchez, Díaz y Lagarda (2017) concluyen que, si bien el proceso formativo y de certificación de competencias en sus profesores ha implicado un trabajo continuo, aún no se logran las metas establecidas, lo que ha complicado determinar en qué grado se implementa innovación docente. Por ello, se establece como un pendiente consolidar las transformaciones de la práctica educativa.

\section{Discusión}

La revisión documental analizada en este artículo aporta una visión integral de los conceptos de innovación como un componente indispensable para la transformación docente, y ofrece un acercamiento conceptual sobre la evaluación del desempeño docente y la certificación de competencias. A partir del análisis documental efectuado, una primera conclusión es: la innovación docente es una de las metas de toda institución educativa y es solo a partir de la inclusión de nuevas formas de trabajo como los profesionales desarrollan aptitudes, habilidades y competencias necesarias para responder a las cambiantes demandas educativas actuales y futuras (Olivera, Sánchez y Marcos, 2015), que exigen una formación de los profesores para la utilización de herramientas de la era digital y transformar su práctica mediante el desarrollo de competencias en el uso de las TIC para promover el aprendizaje (Pinto, Cortés y Alfaro, 2017).

Una segunda conclusión es: la transformación de la práctica docente propia se genera solo a partir de procesos reflexivos en donde el docente consolida sus metodologías y apuntala su propio aprendizaje, identificando cambios específicos que le permiten mejorar continuamente la actividad y la calidad de los componentes que integran el proceso de enseñanza-aprendizaje y, por lo tanto, de los resultados de aprendizaje de sus alumnos (Medina, Hernández y Monsalve, 2015). Para lograr esta meta es imprescindible hacer cambios en los procesos de formación docente, dejar de indicar a los profesores lo que deben hacer; por el contrario, diseñar programas que faciliten el conocimiento profundo sobre sí mismos y motivar una intervención decidida en sus quehaceres (Fandiño-Parra, Ramos-Holguín, Bermúdez-Jiménez y Arenas-Reyes, 2016). 
En tercer lugar, puede concluirse que la evaluación del desempeño docente debe centrarse en la práctica, como una manifestación externa e integradora que le permite recuperar información valiosa sobre su actuación y, al mismo tiempo, recibir la retroalimentación necesaria para promover la reflexión (Vizuete y Muñoz, 2016). La revisión de la teoría sobre la evaluación del proceso educativo indica que la mejora solo se logra si las personas identifican y persiguen sus propios propósitos, pero para ello es necesaria la socialización y el liderazgo académico, la valoración del trabajo entre pares y la comunicación efectiva (Hernández, Tobón y Vázquez, 2015).

Finalmente, se pudo establecer que no hay investigación suficiente sobre los efectos en el rendimiento de los estudiantes o la mejora de los procesos de enseñanza-aprendizaje de docentes certificados, ya que las fuentes analizadas describen casos particulares sobre innovación de la práctica docente, pero no demuestran que estas innovaciones sean producto de la certificación (Kane, Rockoff y Staiger, 2008). Se concluye que es necesario realizar nuevos estudios, ya sea documentales o empíricos, que permitan precisar de qué manera los procesos formativos y la certificación están incidiendo o no en la mejora del aprendizaje.

\section{Referencias}

Álvarez, C.Á. (2015). "De la innovación educativa a la transformación social: Teoría y práctica". Intangible Capital, 11(3), 285-292. doi:10.3926/ic.663

Ausín, V., V. Abella, V. Delgado y D. Hortigüela (2016). "Aprendizaje basado en proyectos a través de las TIC: Una experiencia de innovación docente desde las aulas universitarias". Formación Universitaria, 9(3), 31-38. https://goo.gl/iUixVV

Ávila-Meléndez, L.R. y J.A. Cortés-Montalvo (2017). “La sistematización de experiencias educativas. Una experiencia con docentes universitarios. European Scientific Journal, ESJ, 13(4). https://goo.gl/Gv4bXc

Aznar-Minguet, P., M.A. Ull, M.P. Martínez-Agut y A. Piñero (2017). "Evaluar para transformar: evaluación de la docencia universitaria bajo el prisma de la sostenibilidad". Enseñanza de las Ciencias: Revista de Investigación y Experiencias Didácticas, 35(1), 5-27. https://goo.gl/ o4weao

Bueno-García, C., M. Ubieto-Artur, A.R. Abadía-Valle (2017). “Opinión del estudiantado de la Universidad de Zaragoza sobre las competencias docentes del profesorado universitario". Teoría de la Educación. Educación y Cultura en la Sociedad de la Información, (18), 75-99. https:// goo.gl/WezmeK

Cela-Ranilla, J.M., V. Esteve-González, F. Esteve-Mon, J. González-Martínez y M. Gisbert-Cervera (2017). "El docente en la sociedad digital: una propuesta basada en la pedagogía trans- 
formativa y en la tecnología avanzada". Profesorado. Revista de Currículum y Formación de Profesorado, (21), 403-422. https://goo.gl/STwf5J

Crisol Moya, E. (2017). "Using Active Methodologies: The Student's View". Procedia-Social and Behavioral Sciences, (237), 672-677. https://goo.gl/Qx3HQr

Espinoza de los Monteros, C.J. (2017). Tercer informe de actividades 2015-2016. Sistema de Educación Media Superior. Guadalajara: SEMS. https://goo.gl/DV3DqD

Fandiño-Parra, Y. (2017). “Formación y desarrollo docente en lenguas extranjeras: revisión documental de modelos, perspectivas y políticas". Revista Iberoamericana de Educación Superior, 8(22). https://goo.gl/JsAr7p

B. Ramos-Holguín, J. Bermúdez-Jiménez y J.C. Arenas-Reyes (2016). “Nuevos discursos en la formación docente en lengua materna y extranjera en Colombia". Universidad de La Sabana-Facultad de Educación, 19(1), 46-64. https://goo.gl/Fha25u

Figueroa-Molina, R., C. Salazar-Díaz y M. Bernal-Martínez (2017). “Conocimiento pedagógico en docentes de investigación formativa en la Universidad del Atlántico". Ted: Tecné, Episteme y Didaxis. https://goo.gl/9mqkqm

González-Bernal, V.M., F. Hernández-Guerrero, L.S. Ibarra Sánchez y J.H. del Real Flores (2016). "Modelo de capacitación pedagógica para planear el proceso de enseñanza aprendizaje del nivel superior". Tecnocientifica.com.mx. https://goo.gl/Nf2USS

González Isasi, R.M., C.H. de la Garza Saldívar y M.E. de León Hernández (2017). “Consejos técnicos escolares, un espacio de colaboración para evaluar el ejercicio docente en Educación Básica". Revista Electrónica de Investigación Educativa, 19(3). https://goo.gl/nuyHgb

González Olivares, Á.L. (2014). "Las competencias docentes de la formación para el empleo". Infad.eu. https://goo.gl/qUyM2G

Harvey-López, I.C. (2015). “Evaluación de un modelo de gestión de innovación en la práctica educativa apoyada en las TIC. Estudio de caso: UNIMET". Píxel-Bit. Revista de Medios y Educación, 135-149. doi:10.12795/pixelbit.2015.i47.09

Hernández-Mosqueda, J.S., S. Tobón-Tobón y G. Guerrero-Rosas (2016). “Hacia una evaluación integral del desempeño: las rúbricas socioformativas". Ra Ximhai, 12, 359-376. https://goo. $\mathrm{gl} / \mathrm{cdqsCT}$

Hernández-Jaime, J., Y.I. Jimenez Galán y M. González-Ramírez (2017). “Diseño y validación de un instrumento para explorar el 'saber conocer'y el 'saber hacer' de la evaluación por parte del docente". Pag.org.mx. https://goo.gl/gaF4kU

Hernández, J.S. (2013). "Procesos de evaluación de las competencias desde la socioformación." RaXimhai, 9(4), 11-19. https://goo.gl/j7DCNj

S. Tobón y J.M. Vázquez (2015). "Estudio del liderazgo socioformativo mediante la cartografía conceptual". Revista Iberoamericana de Evaluación Educativa, 8(2), 105-128. https:// goo.gl/wNhXrB 
Hernández-Mosqueda, J., G. Guerrero-Rosas y S. Tobón-Tobón (2015). "Los problemas del contexto: base filosófica y pedagógica de la socioformación". Ra Ximhai, 11(04), 125-140. https://goo.gl/eqepCP

Herrera-Sánchez, S., N.V. Sanchez Sulú, J.J. Díaz-Perera y V. Lagarda-Contreras (2017). “Impacto del Programa de Formación docente del periodo 2011-2016: Caso UNACAR". Ctes.org.mx. https://goo.gl/1U5QEL

Kane, T.J., J.E. Rockoff y D.O. Staiger (2008). "What does Certification Tell us About Teacher Effectiveness? Evidence from New York City". The National Bureau of Economic Research, 27(6), 615-635. https://goo.gl/BEvFZu

Leal-Costa, C., J. Díaz-Agea, A. Rojo-Rojo, L. Juguera-Rodríguez y M. López-Arroyo (2016). “Practicum y simulación clínica en el grado de Enfermería, una experiencia de innovación docente". REDU, Revista de Docencia Universitaria, 12(2), 421-451. https://goo.gl/ErQcPX

León-Guerrero, M.J. y M. del C. López-López (2014), “Criterios para la evaluación de los proyectos de innovación docente universitarios", ESE. Estudios sobre Educación, (26), 79-101. https:// goo.gl/CnktHL

Leyva-López, H.P. (2017). "Impacto del uso de instrumentos de evaluación en el rendimiento académico. Caso estudiantes universitarios de la Licenciatura en Turismo". Revista Iberoamericana de Producción Académica y Gestión Educativa. https://goo.gl/Yw8XoF

López Meneses, E., A. Jaén-Martínez y J. Cabero-Almenara (2013). “Los portafolios educativos virtuales en las aulas universitarias. Instrumentos didácticos para la innovación docente y la calidad de los procesos de enseñanza y aprendizaje". Enseñanza \& Teaching, 31(1), 43-70. https://goo.gl/TPPdRH

Marcelo, C. (2013). "Las tecnologías para la innovación y la práctica docente". Revista Brasileira de Educação, (18), 25-47. https://goo.gl/49LFWs

Margelis-Matos, Y., O.J. Cegarra-Cegarra y C.E. Rivera-Moreno (2017). "La praxis docente desde la formación permanente". Revista Scientific, 2(4), 320-337. https://goo.gl/pLkpwD

Maroto-Molina, F., A. Gómez-Cabrera, A. Garrido-Varo, D.C. Pérez-Marín y E.J.D. Pérez Sanz (2017). "El método del caso como herramienta para la docencia y el aprendizaje en ingeniería de sistemas de producción ganadera". Revista de Innovación y Buenas Prácticas Docentes, (1), 207-216. https://goo.gl/gxKTB4

Medina-Rivulla, A. et al. (2015). Innovación de la educación y de la docencia. Madrid: editorial universitaria Ramón Areces. https://goo.gl/Fjcxvn

Medina-Vidal, F., E. Hernández-Gómez y L. Monsalve-Lorente (2015). “Medios de comunicación utilizados en los centros educativos para difundir los procesos de innovación docente". Revista Lasallista de Investigación, 12(2), 45-53. https://goo.gl/2MBnY4

Morán-Ceja, L.M., A.G. Cortés y K. Vásquez-Suárez (2017). “Evaluación para transformar: Experiencia en la aplicación de proyectos en el preescolar". Debates en Evaluación y Currículum/ 
Congreso Internacional de Educación Evaluación 2016 / Año 2, No. 2, septiembre de 2016 a Agosto de 2017. https://goo.gl/JB1t2d

Muñoz-Serrano, E., C. Yebero-Serrano, J. Muñoz-Espadero y A. Tejero-del Caz (2017). “Innovación docente en las Jornadas de Recepción de Alumnado de ESO y Bachillerato al Departamento de Física". Revista de Innovación y Buenas Prácticas Docentes, (1), 74-82. https://goo. gl/4xUyK2

Muñoz-González, J.M., R. Serrano Rodríguez y V. Marín Díaz (2014). “El aprendizaje colaborativo y su desarrollo a través de mapas mentales. Una innovación educativa en la formación inicial docente". Educatio Siglo XXI, 32(2), 193-212. https://goo.gl/Rf1WT7

Olivera-Zaldua, M., J. Sánchez-Vigil y J.C. Marcos-Recio (2015). “Fotohistoria: modelo de innovación docente". Revista de Sistemas de Información y Documentación, (9), 21-28. https:// goo.gl/9rv31V

Olmos-Alcaraz, A. y R. Martínez-Chicón (2015). "Plataformas virtuales e innovación docente universitaria: Affordance de una Guía de Trabajo Autónomo en Antropología para trabajar competencias de intervención en contextos de diversidad". Revista de Educación a Distancia, 0(42). https://goo.gl/cJFsd9

Ortega-Carbajal, M., J. Hernández-Mosqueda y S. Tobón-Tobón (2015). “Análisis documental de la gestión del conocimiento mediante la cartografía conceptual". Ra Ximhai, 11(4), 141-160. https://goo.gl/5vhyHc

Oviedo-Gonzalez, E., D. Hernández y J.F. Oviedo Villavicencio (2016, enero). "La RIEMS y la formación docente: Retos y oportunidades de frente a la Ley del Servicio Profesional Docente". Congreso Virtual sobre Tecnología, Educación y Sociedad, 1(6). https://goo.gl/ouDVQq

Padilla-Arias, A. (2014). "Innovaciones en la práctica docente universitaria en el ámbito internacional". Argumentos, (27), 105-124. https://goo.gl/UsvkJ5

Palazuelos, E., P. San-Martín, J.M. del Corte y A. Fernández-Laviada (2017). “Utilidad percibida del aprendizaje orientado a proyectos para la formación de competencias. Aplicación en la asignatura 'Auditoría de cuentas'". Revista de Contabilidad. https://goo.gl/KK3XuE

Perez-Vicente, C., et al. (2017). "Uso de mandos interactivos de respuesta como base para la mejora de la docencia en los grados impartidos en la Facultad de Ciencias". Revista de Innovación y Buenas Prácticas Docentes, (1), 42-56. https://goo.gl/tzUk8A

Pierella, M.P. (2017). “Enseñar en la universidad pública argentina: los desafíos del oficio docente en una época de transformaciones". Roteiro, 42(1), 37-64. https://goo.gl/wkAgr8

Pinto Santos, A.R., O. Cortés-Peña y C. Alfaro-Camargo (2017). "Hacia la transformación de la práctica docente: modelo espiral de competencias TICTACTEP". Pixel-Bit. Revista de Medios y Educación, (51), 37-51. https://goo.gl/K6cB48

Romero-López, M.A. (2017). "European Higher Education Area-Driven Educational Innovation”. Procedia-Social and Behavioral Sciences, (237), 1505-1512. https://goo.gl/njEGjv 
Rosario-Muñoz, V.M., D. Elicerio Conchas, O. Zaragoza Vega, G.A. Varela Navarro y M.T. Partida-Sarabia (2016). Innovar para transformar las prácticas docentes. Guadalajara: Amaya Ediciones.

Ruiz, E. (2013). "Reflexión de la práctica docente universitaria desde las estrategias discursivas". Revista Electrónica de Investigación Educativa, 15(1), 88-98. https://goo.gl/HJgdq4

Santiago-Campión, R., F. Navaridas-Nalda y L.A. Andía Celaya (2016). “Las percepciones de los directivos de centros escolares sobre el uso y el valor de las TIC para el cambio e innovación educativa". Estudios sobre Educación, 45-174. doi:10.15581/004.30.145-174

Seixas, P.S., O. Yamamoto, M. Peñaranda-Cólera, A. Garrido-Luque y J.Á Estramiana (2016). “La formación en psicología social de segundo ciclo en el Espacio Europeo de Educación Superior. Quaderns de Psicologia, 18(1), 93-106. https://goo.gl/VJ6fGj

Suárez, D. (2017). “Docentes, relatos de experiencia y saberes pedagógicos. La documentación narrativa de experiencias en la escuela". Investigación Cualitativa, 2(1), 42-54. https://goo. gl/JChKFn

Vázquez-Bernal, B., V. Mellado, R. Jiménez-Pérez y M. Taboada-Leñero (2011). "The Process of Change in a Science Teacher's Professional Development: A Case Study Based on the Types of Problems in the Classroom". doi:10.1002/sce.20474

Veloquio-González, G. (2017). "La formación permanente de los docentes, ante el desafío de atender a la diversidad educativa. Revista de Educación Inclusiva, 9(2). https://goo.gl/zkHiU4

Vera Noriega, J.Á., L.E. Torres Morán y E.E. Martínez-García (2014). “Evaluación de competencias básicas en TIC en docentes de educación superior en méxico". Pixel-Bit. Revista de Medios y Educación, 143-155. https://goo.gl/vv8Uug

Vizuete-Gaibor, L. L. y H. J. Muñoz-Ponce (2016). "La evaluación basada en competencias. Su incidencia en la formación profesional de la educación superior". Revista Electrónica Formación y Calidad Educativa, IV(2), 113-124. https://goo.gl/L4ayBM 